\begin{tabular}{|c|l|}
\hline Title & Essential norms of some singular integral operators \\
\hline Author(s) & Nakazi, T. \\
\hline Citation & Hokkaido University Preprint Series in Mathematics, 462, 1-6 \\
\hline Issue Date & 1999-6-1 \\
\hline DOI & 10.14943/83608 \\
\hline Doc URL & http://hdl.handle.net/2115/69212 \\
\hline Type & bulletin (article) \\
\hline File Information & pre462.pdf \\
\hline
\end{tabular}

Instructions for use 


\title{
Essential Norms Of \\ Some Singular Integral Operators
}

\author{
Takahiko Nakazi
}

Series \# 462. June 1999 


\title{
Essential Norms Of Some Singular Integral Operators
}

by

Takahiko Nakazi*

\author{
Department of Mathematics \\ Faculty of Science \\ Hokkaido University \\ Sapporo 060-0810, Japan
}

nakazi @ math. sci. hokudai. ac jp

*This research was partially supported by Grant-in-Aid for Scientific Research, Ministry of Education

1991 Mathematics Subject Classification. Primary 45 E 10, 47 B 35.

Keywords and phrases. Singular integral operator, essential norm 
Abstract. Let $\alpha$ and $\beta$ be bounded measurable functions on the unit circle $T$. The singular integral operator $S_{\alpha, \beta}$ is defined by $S_{\alpha, \beta} f=\alpha P f+\beta Q f\left(f \in L^{2}(T)\right)$ where $P$ is an analytic projection and $Q$ is a co-analytic projection. In the previous paper, the norm of $S_{\alpha, \beta}$ was calculated in general, using $\alpha, \beta$ and $\alpha \bar{\beta}+H^{\infty}$ where $H^{\infty}$ is a Hardy space in $L^{\infty}(T)$. In this paper, the essential norm $\left\|S_{\alpha, \beta}\right\|_{e}$ of $S_{\alpha, \beta}$ is calculated in general, using $\alpha \bar{\beta}+H^{\infty}+C$ where $C$ is a set of all continuous functions on $T$. Hence if $\alpha \bar{\beta}$ is in $H^{\infty}+C$ then $\left\|S_{\alpha, \beta}\right\|_{e}=\max \left(\|\alpha\|_{\infty},\|\beta\|_{\infty}\right)$. This gives a known result when $\alpha, \beta$ are in $C$. 


\section{$\S 1$. Introduction and results}

Let $m$ denote the normalized Lebesgue measure on the unit circle $T$. For $1 \leq$ $p \leq \infty, L^{p}=L^{p}(T, m)$ denotes the usual Lebesgue space on $T$ and $H^{p}$ denotes the usual Hardy space on $T$. Let $S$ be the singular integral operator defined by

$$
(S f)(\zeta)=\frac{1}{\pi i} \int_{T} \frac{f(\eta)}{\eta-\zeta} d \eta \quad(\text { a.e. } \zeta \in T)
$$

where the integral is understood in the sense of Cauchy's principal value $(c f$. [3, Vol I, p12]). If $f$ is in $L^{1}$ then $(S f)(\zeta)$ exists for almost everwhere $\zeta$ on $T$. Let an analytic projection and a co-analytic projection be

$$
P=(I+S) / 2 \text { and } Q=(I-S) / 2,
$$

where $I$ denotes the identity operator. If $\alpha, \beta \in L^{\infty}$, then the singular integral operator $S_{\alpha, \beta}$ on $L^{2}$ is defined by

$$
S_{\alpha, \beta} f=\alpha P f+\beta Q f \quad\left(f \in L^{2}\right) .
$$

The following inequality is well known and not difficult to establish

$$
\max \left\{\|\alpha\|_{\infty},\|\beta\|_{\infty}\right\} \leq\left\|S_{\alpha, \beta}\right\| \leq\left\|\sqrt{|\alpha|^{2}+|\beta|^{2}}\right\|_{\infty} .
$$

In the previous paper, the author and T.Yamamoto [4] showed the following theorem. This implies that $\left\|S_{\alpha, \beta}\right\|=\max \left(\|\alpha\|_{\infty},\|\beta\|_{\infty}\right)$ when $\alpha \bar{\beta} \in H^{\infty}$.

Norm Theorem. Let $\alpha, \beta \in L^{\infty}$. Then

$$
\left\|S_{\alpha, \beta}\right\|^{2}=\inf _{k \in H^{\infty}}\left\|\frac{|\alpha|^{2}+|\beta|^{2}}{2}+\sqrt{|\alpha \bar{\beta}-k|^{2}+\left(\frac{|\alpha|^{2}-|\beta|^{2}}{2}\right)^{2}}\right\|_{\infty} .
$$

The essential norm $\left\|S_{\alpha, \beta}\right\|_{e}$ is the distance to $\mathcal{K}\left(L^{2}\right)$, the set of all compact operators on $L^{2}$. It is known [3, Vol II, p219] that $\left\|S_{\alpha, \beta}\right\|_{e}=\max \left(\|\alpha\|_{\infty},\|\beta\|_{\infty}\right)$ when $\alpha, \beta$ are in $C$, a set of all continuous functions on $T$. In this paper, we show the following theorem using Norm Theorem. This implies the above result.

Essential Norm Theorem. Let $\alpha, \beta \in L^{\infty}$. Then

$$
\left\|S_{\alpha, \beta}\right\|_{e}^{2}=\inf _{k \in H^{\infty}+C}\left\|\frac{|\alpha|^{2}+|\beta|^{2}}{2}+\sqrt{|\alpha \bar{\beta}-k|^{2}+\left(\frac{|\alpha|^{2}-|\beta|^{2}}{2}\right)^{2}}\right\|_{\infty} .
$$


Corollary. If $\alpha \bar{\beta} \in H^{\infty}+C$, then $\left\|S_{\alpha, \beta}\right\|_{e}=\max \left(\|\alpha\|_{\infty},\|\beta\|_{\infty}\right)$.

The norms and essential norms of Toeplitz operators and Hankel operators are very well known. If $\phi \in L^{\infty}$, then the Toeplitz operator $T_{\phi}$ is defiened by $T_{\phi} f=P(\phi f)$ for $f \in H^{2}$. The Hankel operator $H_{\phi}$ is defined by $H_{\phi} f=Q(\phi f)$ for $f \in H^{2} .\left\|T_{\phi}\right\|=$ $\|\phi\|_{\infty}(c f .[2])$ and $\left\|H_{\phi}\right\|=\left\|\phi+H^{\infty}\right\|$ by the Nehari theorem [6] (cf. [5]). $\left\|T_{\phi}\right\|_{e}=$ $\|\phi\|_{\infty}(c f .[2])$ and $\left\|H_{\phi}\right\|_{e}=\left\|\phi+H^{\infty}+C\right\|(c f .[5])$.

\section{§. The proof of Essential Norm Theorem}

Put $U=S_{z, \bar{z}}$, then $U^{n}=S_{z^{n}, \bar{z}^{n}}$ and $U^{* n} U^{n}=I$ for any positive integer $n$. Let $K$ be arbitrary compact operator on $L^{2}$, that is, $K \in \mathcal{K}\left(L^{2}\right)$. Then

$$
\begin{aligned}
& \left\|S_{\alpha, \beta}+K\right\| \\
& \quad \geq\left\|\left(S_{\alpha, \beta}+K\right) U^{n}\right\| \geq\left\|S_{z^{n} \alpha, \bar{z}^{n} \beta}\right\|-\left\|K U^{n}\right\| \\
& \quad \geq\left\|S_{z^{n}, \bar{z}^{n} \beta}\right\|-\left(\left\|P K U^{n} P\right\|+\left\|P K U^{n} Q\right\|\right. \\
& \left.\quad+\left\|Q K U^{n} P\right\|+\left\|Q K U^{n} Q\right\|\right)
\end{aligned}
$$

Here $P K U^{n} Q=P K Q \bar{z}^{n} Q, Q K U^{n} P=Q K P z^{n} P, P K U^{n} P=P K P z^{n} P$ and $Q K U^{n} Q=$ $Q K Q \bar{z}^{n} Q$. Since $\left(P z^{n} P\right)^{*} \rightarrow 0$ and $\left(Q \bar{z}^{n} Q\right)^{*} \rightarrow 0$ as $n \rightarrow \infty$ in the strong opertor topology.

$$
\lim \left\|P K U^{n} Q\right\|=\lim \left\|Q K U^{n} P\right\|=0 .
$$

and

$$
\lim \left\|P K U^{n} P\right\|=\lim \left\|Q K U^{n} Q\right\|=0
$$

because $P K Q, Q K P, P K P$ and $Q K Q$ are all compact.

By Norm Theorem in $\S 1$,

$$
\left\|S_{z^{n} \alpha, \bar{z}^{n} \beta}\right\|^{2}=\inf _{k \in \bar{z}^{2 n} H^{\infty}}\left\|\frac{|\alpha|^{2}+|\beta|^{2}}{2}+\sqrt{|\alpha \bar{\beta}-k|^{2}+\left(\frac{|\alpha|^{2}-|\beta|^{2}}{2}\right)^{2}}\right\|_{\infty}
$$

and hence

$$
\begin{aligned}
& \lim _{n \rightarrow \infty}\left\|S_{\bar{z}^{n} \alpha, \bar{z}^{n} \beta}\right\|^{2} \\
& =\inf _{k \in H^{\infty}+C}\left\|\frac{|\alpha|^{2}+|\beta|^{2}}{2}+\sqrt{|\alpha \bar{\beta}-k|^{2}+\left(\frac{|\alpha|^{2}-|\beta|^{2}}{2}\right)^{2}}\right\|_{\infty}
\end{aligned}
$$


because $H^{\infty}+C$ is closed. Thus

$$
\left\|S_{\alpha, \beta}\right\|_{e}^{2} \geq \inf _{k \in H^{\infty}+C}\left\|\frac{|\alpha|^{2}+|\beta|^{2}}{2}+\sqrt{|\alpha \bar{\beta}-k|^{2}+\left(\frac{|\alpha|^{2}-|\beta|^{2}}{2}\right)^{2}}\right\|_{\infty}
$$

Put $P_{1}=z P \bar{z}$, then $P_{1}$ is an orthogonal projection from $L^{2}$ to $z H^{2}$ and $P_{1}=$ $z P \bar{z}(P+Q)=z P \bar{z} P$. Simitarly, $Q_{1}=\bar{z} Q z$ is an orthogonal projection from $L^{2}$ to $\bar{z}^{2} \bar{H}^{2}$ and $Q_{1}=\bar{z} Q z Q$. Since $P-P_{1}$ and $Q-Q_{1}$ are rank one projections, $\|\alpha P+\beta Q\|_{e}=$ $\left\|\alpha P_{1}+\beta Q_{1}\right\|_{e}$. Since $\beta \bar{z} Q \bar{z} P$ and $\alpha z P z Q$ are finite rank operators,

$$
\begin{aligned}
& \|(\alpha z P+\beta \bar{z} Q)(\bar{z} P+z Q)\|_{e} \\
& \quad=\|\alpha z P \bar{z} P+\beta \bar{z} Q z Q+\beta \bar{z} Q \bar{z} P+\alpha z P z Q\|_{e} \\
& =\left\|\alpha P_{1}+\beta Q_{1}\right\|_{e}=\|\alpha P+\beta Q\| .
\end{aligned}
$$

Hence

$$
\begin{aligned}
\left\|S_{\alpha, \beta}\right\|_{e} & \leq\left\|S_{z \alpha, \bar{z} \beta}\right\|_{e}\|\bar{z} P+z Q\|_{e} \\
& \leq\left\|S_{z \alpha, \bar{z} \beta}\right\|_{e} \\
& =\left\|S_{z \alpha, \bar{z} \beta}\right\|
\end{aligned}
$$

because

$$
\begin{aligned}
\|\bar{z} P+z Q\|_{e} & =\|P \bar{z} P+Q z Q\|_{e} \\
& =\max \left\{\|P \bar{z} P\|_{e},\|Q z Q\|_{e}\right\}=1
\end{aligned}
$$

Therefore

$$
\begin{aligned}
\left\|S_{\alpha, \beta}\right\|_{e} & \leq \lim _{n \rightarrow \infty}\left\|S_{z^{n} \alpha, \bar{z}^{n} \beta}\right\| \\
& =\inf _{k \in H^{\infty}+C}\left\|\frac{|\alpha|^{2}+|\beta|^{2}}{2}+\sqrt{|\alpha \bar{\beta}-k|^{2}+\left(\frac{|\alpha|^{2}-|\beta|^{2}}{2}\right)^{2}}\right\|_{\infty} .
\end{aligned}
$$

This completes the proof of Essential Norm Theorem. 


\section{References}

1. S.Axler, I.D.Berg, N.Jewell, and A.Shields, Approximation by compact operators and the space $H^{\infty}+C$, Ann. of Math. 109(1979), 601-612.

2. R.G.Douglas, Banach Algebra Techniques in Operator Theory, Academic Press, New York, 1972.

3. I.Gohberg and N.Krupnik, One-dimensional linear singular integral equations, Vols. I,II, Birkhäuser Verlag, Basel, 1992.

4. T.Nakazi and T.Yamamoto, Norms of some singular integral operators and their inverse operators, J.Operator Th. 40(1998), 185-207.

5. N.K.Nikol'skii, Treatise on the Shift Operator, Springer Verlag, Berlin, 1986.

6. Z.Nehari, On bounded bilinear forms, Ann. of Math. 65(1957), 153-162. 